\title{
Role of Bulk and Surface Phonons in the Decay of Metal Surface States
}

\author{
A. Eiguren, ${ }^{1}$ B. Hellsing, ${ }^{2}$ F. Reinert,${ }^{3}$ G. Nicolay, ${ }^{3}$ E. V. Chulkov, ${ }^{1,4}$ V. M. Silkin, ${ }^{4}$ S. Hüfner, ${ }^{3}$ and P. M. Echenique ${ }^{1,4}$ \\ ${ }^{1}$ Departmento de Física de Materiales and Centro Mixto CSIC-UPV/EHU, Facultad de Ciencias Químicas, \\ Universidad del Pais Vasco/Euskal Herriko Unibertsitatea, Apdo. 1072, 20018 San Sebastián/Donostia, Basque Country, Spain \\ ${ }^{2}$ Department of Physics, Chalmers University of Technology and Göteborg University, S-412 96 Göteborg, Sweden \\ ${ }^{3}$ Fachrichtung Experimentalphysik, Universität des Saarlandes, 66041 Saarbrücken, Germany \\ ${ }^{4}$ Donostia International Physics Center (DIPC), Paseo de Manuel Lardizabal, 4, 20018 San Sebastián/Donostia, Spain
}

(Received 30 October 2001; published 28 January 2002)

\begin{abstract}
We present a comprehensive theoretical investigation of the electron-phonon contribution to the lifetime broadening of the surface states on $\mathrm{Cu}(111)$ and $\mathrm{Ag}(111)$, in comparison with high-resolution photoemission results. The calculations, including electron and phonon states of the bulk and the surface, resolve the relative importance of the Rayleigh mode, being dominant for the lifetime at small hole binding energies. Including the electron-electron interaction, the theoretical results are in excellent agreement with the measured binding energy and temperature dependent lifetime broadening.
\end{abstract}

DOI: $10.1103 /$ PhysRevLett.88.066805

PACS numbers: 73.20.At, 71.38.-k, 79.60.Bm

Understanding the temporal evolution of quasiparticles (electron and holes) on metal surfaces is of paramount importance to describe many important phenomena such as the dynamics of charge and energy transfer, quantum interference, localization, and many others. This temporal evolution is characterized by a finite lifetime, $\tau$, which refers to the time the quasiparticle retains its identity. While the lifetime of an excited electron or hole is determined by many-body interactions, namely, electronelectron $(e-e)$ and electron-phonon $(e-p)$ scattering processes, the peak width in an experiment might also be influenced by electron-defect scattering on crystal or surface imperfections [1]. However, it was demonstrated in recent scanning tunneling microscopy (STM) [2] and photoemission experiments [3] that these defect contributions can be minimized, making it possible to analyze the pure lifetime broadening due to the formation of a hole at the band minimum of the $s p$ surface state band in the $L$ gap of the (111) surface of noble metals.

These Shockley-type surface states form a twodimensional (2D) electron gas and the $e-e$ contribution to the hole lifetime has been rationalized in terms of a dominant contribution from intraband transitions within the 2D surface state band, screened by the underlying 3D bulk electron system, and in terms of interband transitions (surface states $\rightarrow$ bulk states) [2]. On the other hand, an appropriate calculation of the $e-p$ contribution to the lifetime broadening of surface states is still lacking. This paper is an attempt in this direction.

The strength of the $e-p$ coupling shows up in the electron mass enhancement $m^{*}=m(1+\lambda)$, in terms of the parameter $\lambda$, which in general is energy and momentum dependent. Many properties of metals, such as resistivity, specific heat, and superconductivity, reflect the $e-p$ coupling and can be expressed in terms of the Fermi surface-averaged $\lambda$ value [4]. $\lambda$ also reflects the high-temperature behavior of the state broadening $\Gamma_{e p}=2 \pi \lambda k_{B} T$. The anisotropy of $\lambda$ is well known [5] and is revealed in, e.g., cyclotron resonance measurements [6].

Typically, the phonon contribution to the decay of surface states is estimated using the Debye phonon model. Within this model the Eliashberg spectral function $\alpha^{2} F(\omega)$ of the $e-p$ interaction is proportional to the quadratic density of phonon states $\alpha^{2} F(\omega)=\lambda\left(\omega / \omega_{D}\right)^{2}$, where $\omega_{D}$ is the Debye energy and $\lambda$ is usually obtained from measurements or theoretical calculations of bulk properties [4]. However, it is not obvious that this approach should be adequate for surface state electrons or holes when the surface state itself, as well as the surface phonon modes, is not taken into account and, furthermore, the low-temperature $\Gamma_{e p}$ will depend on the model used. A more rigorous treatment of the $e-p$ contribution is needed especially for surface states close to the Fermi level, because for these states the $e-e$ contribution is small and the $e-p$ interaction becomes dominant even at low temperatures.

In this Letter we present a theoretical analysis of the $e-p$ coupling contribution to the lifetime broadening of surface electron states, taking into account all electron and phonon states involved in the electron-phonon scattering process, and compare it with new energy and temperature dependent high-resolution photoemission data. The theoretical analysis is based on a calculation of the full Eliashberg spectral function. This approach enables us to resolve, in detail, the contributions from different phonon modes, in particular the Rayleigh surface mode and bulk phonons and the general temperature dependence. We also obtain the high-temperature behavior represented by $\lambda$, which is given by the first reciprocal moment of the Eliashberg function. We show that an approach based on (i) Thomas-Fermi screened Ashcroft electron-ion potentials, (ii) one-electron states with first principles quality, and (iii) a simple phonon model calculation gives results in good agreement with recently published experimental data $[1-3,7]$ and the new data presented here. 
The phonon-induced lifetime broadening of a surface band state with momentum $\vec{k}_{i}$ and binding energy $\epsilon_{\vec{k}_{i}}$ is given by

$$
\begin{aligned}
\Gamma_{e p}\left(\epsilon_{\vec{k}_{i}}\right)=2 \pi \int_{0}^{\omega_{m}}\left\{\alpha^{2} F_{\vec{k}_{i}}(\omega)[1+2 n(\omega)\right. \\
\left.\left.+f\left(\epsilon_{\vec{k}_{i}}+\omega\right)-f\left(\epsilon_{\vec{k}_{i}}-\omega\right)\right]\right\} d \omega,
\end{aligned}
$$

where $\omega_{m}$ is the maximum phonon frequency and

$$
\alpha^{2} F_{\vec{k}_{i}}(\omega)=\sum_{\vec{q}, \nu, f}\left|g_{i, f}^{\vec{q}, \nu}\right|^{2} \delta\left(\omega-\omega_{\vec{q}, \nu}\right) \delta\left(\epsilon_{\vec{k}_{i}}-\epsilon_{\vec{k}_{f}}\right),
$$

where the sum is over final electron states $\vec{k}_{f}$ of band $f$ and phonon modes $\nu$ with momentum $\vec{q}$.

In Eqs. (1) and (2) we employ the commonly used quasielastic scattering approximation $\delta\left(\epsilon_{\vec{k}_{i}}-\right.$ $\left.\epsilon_{\vec{k}_{f}} \pm \omega_{\vec{q}, \nu}\right) \approx \delta\left(\epsilon_{\vec{k}_{i}}-\epsilon_{\vec{k}_{f}}\right)$. This approximation allows us to calculate $\Gamma_{e p}$ with the same Eliashberg function [Eq. (2)] for both emission and absorption processes. The $e-p$ coupling constant is given by

$$
g_{i, f}^{\vec{q}, \nu}=\sqrt{\frac{1}{2 M N \omega_{\vec{q} \nu}}} \times\left\langle f\left|\sum_{\mu} \vec{\epsilon}_{\vec{q} \nu}\left(\vec{R}_{\mu}\right) \cdot \vec{\nabla}_{\vec{R}_{\mu}} \tilde{V}_{q}^{\mu}\right| i\right\rangle,
$$

summing over the layers $\mu$ of the slab. $M$ and $N$ are the atomic mass and number of ions in each layer of the slab, respectively, $\vec{\epsilon}_{\vec{q}, \nu}\left(\vec{R}_{\mu}\right)$ denotes the complex phonon polarization vectors normalized over the slab, and $\tilde{V}_{q}^{\mu}$ is the screened electron-ion potential. The electron states obtained from a slab calculation are of the form $\psi_{n, \vec{k}}(\vec{x}, z)=$ $\langle z \mid n\rangle \exp (-i \vec{k} \cdot \vec{x})$ where $n$ is the band index, and $\vec{k}$ and $\vec{x}$ are the electron momentum and electron coordinate parallel to the surface, respectively. The slab model potential scheme by Chulkov and co-workers [8-10] gives wave functions which are in good agreement with ab initio calculations and energy spectra reproduce experimental data. We want to point out that, within $\sim 1 \mathrm{eV}$ below the Fermi level, the band structure is known to be nearly free electronlike and no additional surface states are present in the surface Brillouin zone (SBZ) [11]. The slight nonparabolic electron bands near the $\bar{M}$ point of the SBZ is of no importance as the corresponding large momentum scattering processes give negligible contributions to the Eliashberg function in Eq. (2).

The umklapp processes can be neglected since the Fermi momentum of the noble metal surface states is small $(<0.12$ a.u. $)$ in comparison with half the minimum reciprocal vector $(<0.75$ a.u. $)$. Furthermore, the maximum Fermi momentum vector of final bulk states is $<0.74$ a.u., which implies that final states are all confined to the first SBZ. Thus, the contribution from umklapp processes is negligible for small binding energies and vanishes completely when approaching the $\bar{\Gamma}$ point. To obtain the phonon dispersion relations and polarization vectors we perform a calculation using the force constant model by Black et al. [12], and in addition we expand the dynamic matrix in Gottlieb polynomials [13] in order to optimize the representation of the surface phonon modes. The force constants are fitted to reproduce elastic constants and bulk phonon frequencies, with surprisingly good agreement with He scattering experiments [14].

The screened electron-ion potential is determined by the static dielectric function and the bare pseudopotential, $\tilde{V}_{q}^{\mu}(z)=\int d z^{\prime} \tilde{\boldsymbol{\epsilon}}^{-1}\left(z, z^{\prime} ; q\right) \tilde{V}_{\text {bare }}^{\mu}\left(z^{\prime} ; q\right)$, where $q$ is the modulus of the phonon momentum wave vector parallel to the surface and $\tilde{V}_{\text {bare }}^{\mu}$ is the 2D Fourier transform of the bare electron-ion pseudopotential. We use the spherically symmetric Ashcroft pseudopotentials with the single empty-core radii $R=0.813$ and 1.04 a.u. for $\mathrm{Cu}$ and $\mathrm{Ag}$, respectively [15]. The quality of the screening was investigated using the dielectric function according to both Thomas-Fermi and RPA (constructed from the eigen wave functions and eigen energies of a 31-slab calculation). The difference is about $1 \%$ for both $\lambda$ and $\Gamma_{e p}$ due to a compensating effect. Referring to the surface layer, Thomas Fermi screening is symmetric while RPA yields a screening slightly stronger below and slightly weaker above the layer.

We want to point out that, in almost all investigations of the $e-p$ interaction, the relevant electron scattering takes place close to the Fermi surface and only the $e-p$ coupling averaged over the Fermi surface comes into play. When considering the lifetimes of surface states, the situation is different. The $e-p$ coupling becomes state dependent when the probed initial electron state is fixed. For the surface states of the studied noble metal surfaces $\mathrm{Cu}(111)$ and $\operatorname{Ag}(111)$, the Fermi surface is of importance because the binding energy of the initial hole state ranges from 0 (Fermi level) to about $0.4 \mathrm{eV}$.

The intraband electron scattering $(f=i)$ in Eq. (3) can be neglected with the following argument. In the case of intraband transitions in the long wavelength limit, the matrix element of the gradient of the screened electron-ion potential is approximately the expectation value of the force acting on the ions in the direction perpendicular to the surface $\left\langle\psi_{i, \vec{k}}(\vec{x}, z) \nabla_{\vec{R}_{\mu}} \tilde{V}^{\mu}(\vec{x}, z) \psi_{i, \vec{k}-\vec{q}}(\vec{x}, z)\right\rangle \approx$ $\vec{F}_{\mu} \approx\left\langle i\left|\partial \tilde{V}_{0}^{\mu}(z) / \partial z\right| i\right\rangle \hat{z}$. It is well known that, within linear response, the sum over the forces acting on the ions induced by the electron (hole) must be zero [16-19], thus the matrix element of the sum of gradients of the screened electron ion must be equal to zero, $\sum_{\mu} \vec{F}_{\mu}=0$. Furthermore, the ion displacements forming the phonon modes in the surface region, associated with small $q$, are locally rigid with a coherence length of $2 \pi / q$ isotropically in all directions, parallel and perpendicular to the surface [20]. From Eqs. (1)-(3), it can be seen that this restriction and the reduced phase space in the intraband scattering process makes this contribution negligible compared to interband contributions.

From Eq. (2) we see that the Eliashberg function is given by the phonon density of states weighted by the 
electron-phonon coupling strength $g$. In Fig. 1 the calculated phonon dispersion and the Eliashberg function is presented for $\mathrm{Cu}(111)$. The Rayleigh surface mode is split from the bulk phonon band, which gives a lower energy peak in the Eliashberg function, at $\sim 13 \mathrm{meV}$ in $\mathrm{Cu}(111)$. The oscillations in the Eliashberg function reflect the finite number of layers of the model potential (31 layers) in the calculation of electron wave functions and thus have no physical significance.

The high-resolution photoemission experiments presented here were performed with an energy and an angular resolution of $\Delta E \approx 3 \mathrm{meV}$ and $\Delta \Theta \approx 0.3^{\circ}$, respectively, at a vacuum ultraviolet photon energy of $h \nu=21.23 \mathrm{eV}$ (He I). A detailed description of experimental conditions and sample preparation can be found in Ref. [3]. A complete data set at one temperature, consisting of the photoemission data over an angular range of $\pm 6^{\circ}$ off normal, was measured within the first $15 \mathrm{~min}$ after the last annealing step at $\approx 600^{\circ} \mathrm{C}$. As an example, the inset of Fig. 2 demonstrates the temperature dependence of the normal emission ( $\bar{\Gamma}$ point) spectra on $\mathrm{Cu}(111)$ at three different temperatures. For a quantitative analysis we fitted the individual spectra of a temperature series, as described in Ref. [3]. The resulting Lorentzian lifetime widths $\Gamma$ (full widths at half maximum) are given as open symbols in Fig. 2. The binding energy dependence of the linewidth at low temperature (cf. Fig. 3) was determined from momentum distribution curve cuts at the respective energy and are given relative to the linewidth at the Fermi level $E_{F}$, which is nearly completely determined by the finite angular resolution.

The main signature of the $e-p$ contribution to the lifetime broadening is the temperature dependence. For binding energies exceeding $k_{B} T$ we can neglect the temperature

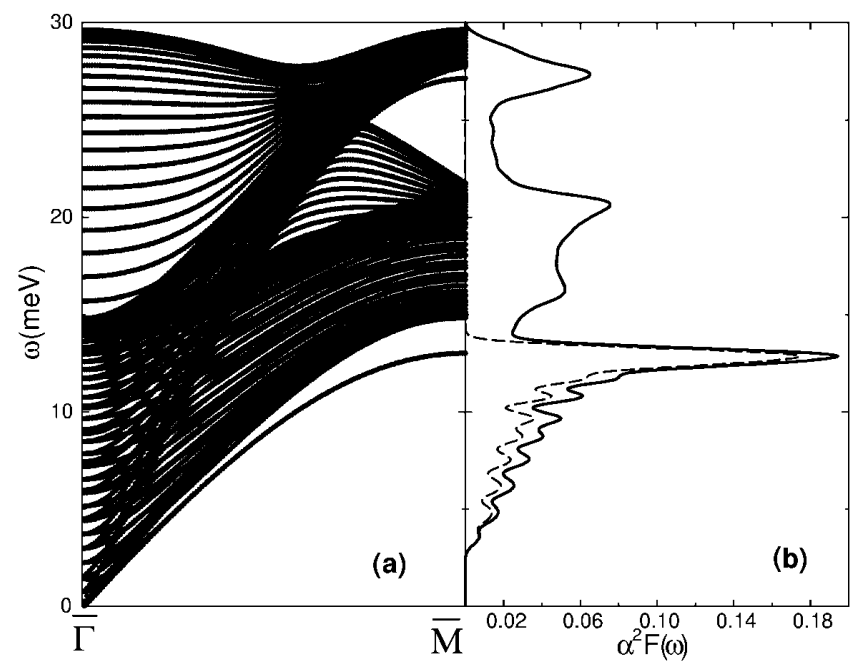

FIG. 1. (a) The phonon dispersion from a 31 layer slab calculation in the $\bar{\Gamma}-\bar{M}$ direction of the SBZ. (b) The Eliashberg function of the hole state in the $\bar{\Gamma}$ point (solid line) and the contribution from the Rayleigh mode to the Eliashberg function (dashed line). dependence of the $e-e$ scattering. The temperature dependence of $\Gamma_{e p}$ was calculated for the hole state in the $\bar{\Gamma}$ point for $\mathrm{Cu}(111)$ and $\mathrm{Ag}(111)$. We have not considered the small thermal effect on the band structure, reflected in the inset of Fig. 2, in the calculation of $\Gamma_{e p}$. However, the small binding energy dependence of the Eliashberg function indicates that this is a higher order effect of minor importance for $\Gamma_{e p}$.

To compare with experimental temperature dependence we show in Fig. 2 the sum of the constant contribution from $\Gamma_{e e}$ and the $T$ dependent $\Gamma_{e p}$. The calculated broadenings agree well with the experimental data points, except for the highest temperature data point for $\mathrm{Cu}(111)$. In Table I we summarize the calculated and measured low-temperature results for the lifetime broadening for the surface states of $\mathrm{Cu}(111)$ and $\mathrm{Ag}(111)$. The sum of the calculated contribution from $e-e$ interaction $\Gamma_{e e}$ [2] and the results of this paper for the $e-p$ interaction $\Gamma_{e p}$ show the proper trend in comparison with both STM [2] and angle-resolved ultraviolet photoemission spectroscopy (ARUPS) [3] data.

The most interesting hole binding energy region is when it is less than the maximum phonon frequency $\omega_{m}$. In this region we expect that the lifetime broadening is completely determined by the $e-p$ coupling. The contribution from $e-e$ interaction is very small, for $\mathrm{Cu}(111)$ and $\operatorname{Ag}(111)$, $\Gamma_{e e}<0.2 \mathrm{meV}$. In Fig. 3 we present the calculated $\Gamma_{e p}$ at

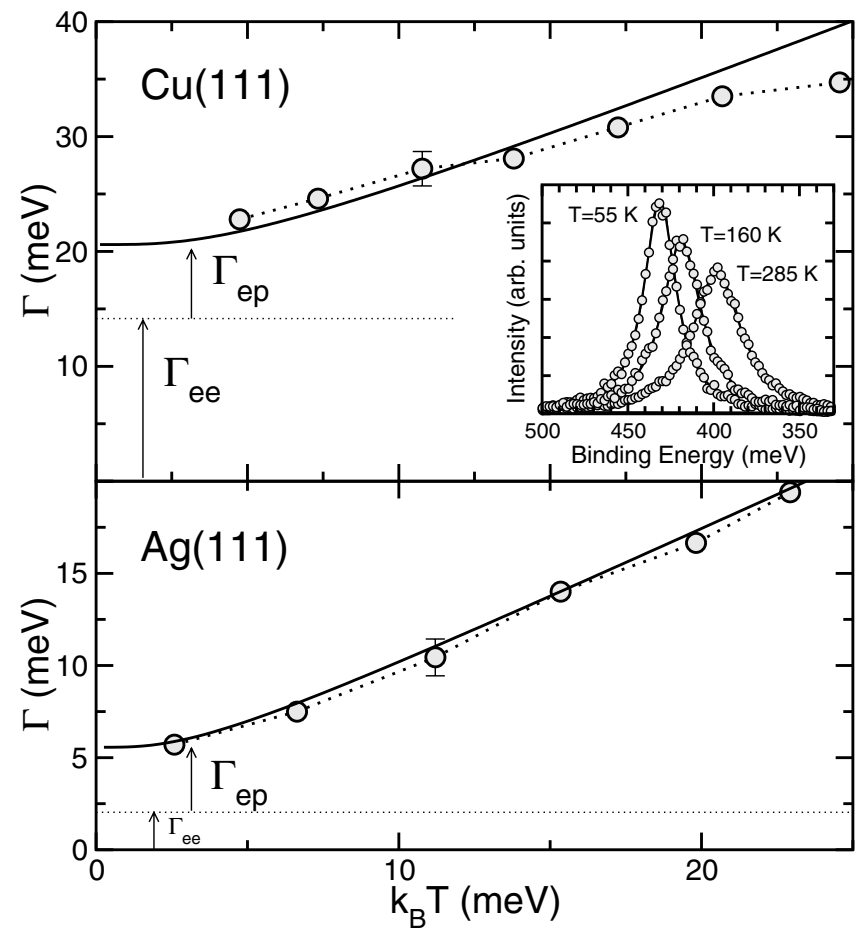

FIG. 2. Calculated lifetime broadening of the $\mathrm{Cu}(111)$ and $\mathrm{Ag}(111)$ surface hole state at the band minimum as a function of temperature (solid lines), $\Gamma_{e e}$ (dotted lines) photoemission data (open circles). The inset shows the energy distribution curves on $\mathrm{Cu}(111)$ for selected temperatures. 


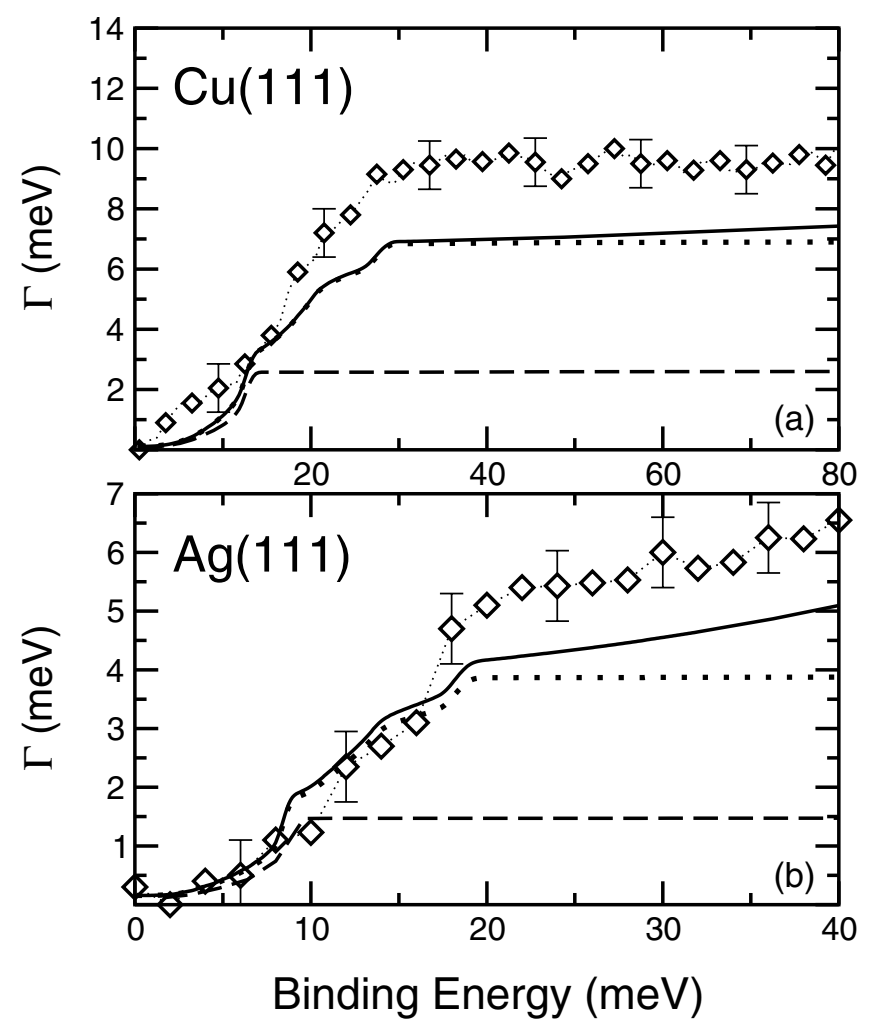

FIG. 3. (a) Lifetime broadening of the $\mathrm{Cu}(111)$ surface hole state as a function of binding energy, $\Gamma_{e e}+\Gamma_{e p}$ (solid line), $\Gamma_{e p}$ (dotted line), the Rayleigh mode contribution to $\Gamma_{e p}$ (dashed line), and photoemission data (diamonds). (b) The same as in (a) for $\operatorname{Ag}(111)$.

$T=30 \mathrm{~K}$, for $\mathrm{Cu}(111)$ and $\mathrm{Ag}(111)$, together with the experimental results. From a simple Debye model we would expect a cubic binding energy dependence in the region below $\omega_{m}$, which obviously is not the case. The saturation of $\Gamma_{e p}$ for binding energies exceeding $\omega_{m}$ is clearly seen in the experimental data. By adding the contribution from the $e$-e interaction, the values close to the experiment are obtained (see Table I). We note from Fig. 3 that the contribution from only the Rayleigh mode gives $\sim 40 \%$ of $\Gamma_{e p}$ beyond the maximum phonon frequencies, and the contribution from bulk and other surface phonon modes gives $\sim 60 \%$. But, for binding energies below the maximum of the Rayleigh mode energy, this mode alone represents, on average, about the $85 \%$ of $\Gamma_{e p}$.

In summary we have presented results from a theoretical and an experimental study of the inherent phonon-induced lifetime broadening of surface states of the noble metal surfaces $\mathrm{Cu}(111)$ and $\mathrm{Ag}(111)$. Taking into account electron and phonon states, including surface states, is crucial for the understanding of experimental findings, in particular, for states close to the Fermi level. The Rayleigh surface phonons are shown to give important contributions to the phonon-induced lifetime broadening, in particular for small binding energies. We have demonstrated that, for noble metal surface states, simple Thomas-Fermi screen-
TABLE I. Calculated and measured low-temperature lifetime widths $\Gamma$ at band minimum in $\mathrm{meV}$ and mass enhancement factors $\lambda$ for the surface states of $\mathrm{Cu}(111)$ and $\operatorname{Ag}(111) . \quad \Gamma_{e e}$ is the calculated lifetime width due to electron-electron scattering [2], $\Gamma_{e p}$ is from electron-phonon scattering (present calculation), $\Gamma_{\mathrm{STM}}$ is from STM measurements at $T=4.6 \mathrm{~K}$ [2], and $\Gamma_{\text {ARUPS }}$ is from ARUPS measurements [3].

\begin{tabular}{lrrcrcc}
\hline \hline & $\Gamma_{e p}$ & $\Gamma_{e e}{ }^{\mathrm{a}}$ & $\Gamma_{e e}+\Gamma_{e p}$ & $\Gamma_{\mathrm{STM}}$ & $\Gamma_{\text {ARUPS }}$ & $\lambda$ \\
\hline $\mathrm{Cu}(111)$ & 6.9 & 14.0 & 21 & 24 & $23 \pm 1.0$ & 0.16 \\
$\mathrm{Ag}(111)$ & 3.9 & 2.0 & 5.9 & 6 & $6 \pm 0.5$ & 0.12 \\
\hline \hline
\end{tabular}

${ }^{\mathrm{a}}$ This value for $\Gamma_{e e}$ is a lower limit, because the used model [2] slightly overestimates the $d$-band effect [21].

ing of Ashcroft pseudopotentials gives very similar results as a more rigorous treatment of the screening. We find excellent agreement with experiments with respect to both binding energy and temperature dependence of the lifetime broadening.

We acknowledge financial support from the Basque Government, the Deutsche Forschungsgemeinschaft (SFB 277), the Carl Tryggers Foundation and the Max Planck Research Award Funds.

[1] F. Theilmann et al., Phys. Rev. B 56, 3632 (1997).

[2] J. Kliewer et al., Science 288, 1399 (2000).

[3] F. Reinert et al., Phys. Rev. B 63, 115415 (2001).

[4] G. Grimvall, in The Electron-Phonon Interaction in Metals, Selected Topics in Solid State Physics, edited by E. Wohlfarth (North-Holland, New York, 1981).

[5] F. S. Khan et al., Phys. Rev. B 26, 1538 (1982).

[6] M. J. G. Lee, Phys. Rev. B 2, 250 (1970).

[7] B. A. McDougall, T. Balasubramanian, and E. Jensen, Phys. Rev. B 51, 13891 (1995).

[8] E. V. Chulkov, V. M. Silkin, and P. M. Echenique, Surf. Sci. 437, 330 (1999).

[9] E. V. Chulkov et al., Phys. Rev. Lett. 80, 4947 (1998).

[10] P. M. Echenique et al., Chem. Phys. 251, 1 (2000).

[11] A. Euceda, D. M. Bylander, and L. Kleinman, Phys. Rev. B 28, 528 (1983).

[12] J. E. Black, F. C. Shanes, and R. F. Wallis, Surf. Sci 133, 199 (1983).

[13] S. Trullinger, J. Math. Phys. 17, 1884 (1976).

[14] U. Harten, J. P. Toennies, and Ch. Wöll, Faraday Discuss. Chem. Soc. 80, 137 (1985).

[15] N. W. Ashcroft and D. C. Langreth, Phys. Rev. 159, 500 (1966).

[16] R. S. Sorbello and B. B. Dasgupta, Phys. Rev. B 21, 2196 (1980).

[17] H.F. Budd and J. Vannimenus, Phys. Rev. B 12, 509 (1975).

[18] S. Andersson et al., Phys. Rev. Lett. 23, 2073 (1984).

[19] M. Persson, J. A. Stroscio, and W. Ho, Phys. Scr. 36, 548 (1987).

[20] M. E. Flatté and W. Kohn, Phys. Rev. B 43, 7422 (1991).

[21] A. García-Lekue et al. (to be published). 